\title{
Validación de un instrumento que mide el perfil actitudinal de los docentes y el desarrollo de competencias universitarias y transversales
}

Validation of an instrument, which measures the teacher's attitudinal profile and the development of university and transversal competencies

Validação de um instrumento que mede o perfil atitudinal de professores $e$ o desenvolvimento universitário e de competências transversais

Espinosa-Solís Josué Israel Universidad Autónoma de Chihuahua, México

jiespinosa@uach.mx https://orcid.org/0000-0002-2808-8038

Pizarro-Norma

Universidad Autónoma de Chihuahua, México

npizarro@uach.mx https://orcid.org/0000-0002-4748-4271

Parra-Acosta Haydeé

Universidad Autónoma de Chihuahua, México hparra05@hotmail.com https://orcid.org/0000-0002-6964-4205

González-Carrillo Eliazar Universidad Autónoma de Chihuahua, México egonzales@uach.mx https://orcid.org/0000-0002-7571-7570

Talavera-Sánchez Oscar Joel Universidad Autónoma de Chihuahua, México otalavera@uach.mx https://orcid.org/0000-0002-4881-4658 
Bueno-Acuña Gerardo

Universidad Autónoma de Chihuahua, México

gbueno@uach.mx

https://orcid.org/0000-0003-1345-9876

\section{Resumen}

En el presente artículo se describen los resultados de la validación de un instrumento que mide el perfil actitudinal de los docentes y el desarrollo de competencias universitarias y transversales, iniciativa que surgió debido a que no se halló un instrumento específico para evaluar de forma integrada las actitudes del docente y las competencias de los estudiantes de la carrera Odontología. Metodológicamente, se planteó un estudio cuantitativo que se desarrolló por procedimiento deductivo. Para ello, se diseñaron dos instrumentos dirigidos a docentes y estudiantes que comprendieron un total de 117 y 118 ítems. Para la validación de contenido se empleó el juicio de expertos, los cuales valoraron su univocidad y pertinencia con base en Carrera, Vaquero y Balsells (2011). La validación de consistencia interna fue mediante el alfa de Cronbach, según las indicaciones de Hernández Sampieri y Mendoza (2018). En los resultados se observó, de acuerdo con los índices de univocidad (IU) y pertinencia (IP), que 109 ítems conservaron su redacción original, ocho fueron modificados y tres se eliminaron. El resultado de confiabilidad del cuestionario fue $\alpha=.972$ para el cuestionario de docentes y $\alpha=.989$ para el de estudiantes. Por ende, se puede concluir que se consiguió un instrumento de medición con alto nivel de confiabilidad, lo que garantiza que la información recabada se pueda generalizar a la población objetivo.

Palabras clave: instrumento, juicio de expertos, perfil actitudinal, validación. 


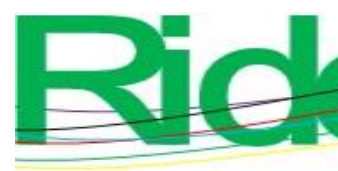

Revista Iberoamericana para la Investigación y el Desarrollo Educativo ISSN 2007 - 7467

$\alpha=0,989$ para o questionário do aluno. Portanto, pode-se concluir que foi alcançado um instrumento de medida com alto nível de confiabilidade, o que garante que as informações coletadas possam ser generalizadas para a população-alvo.

Palavras-chave: instrumento, julgamento de especialistas, perfil atitudinal, validação.

Fecha Recepción: Enero 2021

Fecha Aceptación: Agosto 2021

\section{Introducción}

El profesor universitario en su perfil profesional debe contar no solo con conocimientos, actitudes y valores dentro de su disciplina, sino también con herramientas pedagógicas que sustenten su quehacer docente. Al respecto, Valerio y Rodríguez (2017) definen el perfil actitudinal del docente como los atributos que posee para guiar el aprendizaje esencial en la práctica educativa. En este sentido, cabe mencionar las competencias que Fierro et al. (2019) plantean en el nuevo modelo educativo denominado renovación universitaria UACH-DS 2020. Dentro este, y en proceso de transición del plan de estudios vigente, Fierro et al. (2019) señalan que en el nuevo modelo educativo se socializa en las unidades académicas las competencias universitarias y transversales que todo estudiante debe desarrollar, así como los desempeños que deberán mostrar durante su proceso de aprendizaje.

Para ello, la labor del docente es esencial, pues este profesional debe procurar fomentar en el alumno el dominio de múltiples competencias. Por eso, resulta vital validar el contenido y la consistencia interna de un instrumento que sirva para medir el perfil actitudinal de los docentes y su relación con el desarrollo de las competencias universitarias (en este caso en los estudiantes de la Facultad de Odontología), las cuales se describen a continuación:

- Razonamiento formal y cuantitativo para explicar, modelar y predecir realidades cotidianas sociales, económicas y naturales.

- Investigación y análisis científico para problematizar la realidad natural en diversos contextos a través de observaciones empíricas rigurosas con el fin de responder a preguntas de causa y efecto, formular y probar hipótesis mediante la experimentación e interpretación de datos para hacer inferencias válidas, reconociendo los alcances y limitaciones del conocimiento.

- Investigación y análisis social e histórico para reconocer diferentes formas de organización social, política, económica y cultural, y comprender el comportamiento humano de forma significativa en su contexto y tiempo. 


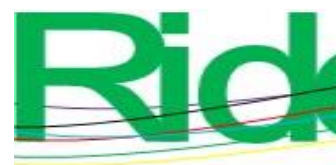

Revista Iberoamericana para la Investigación y el Desarrollo Educativo ISSN 2007-7467

- Multiculturalidad y sociedad para lograr abordar los problemas desde diferentes perspectivas culturales, experiencias históricas y sociales para integrar visiones del mundo comprensivas y tolerantes, respetando e integrando la diversidad.

- Apreciación estética e interpretativa para el reconocimiento del valor estético e interpretación de trabajos y productos culturales.

- Creatividad, innovación y emprendimiento para generar cambios en los sectores económicos, sociales y culturales, a través de la implementación de una respuesta original a una situación crítica y en condiciones de incertidumbre que garantice sostenibilidad (Fierro et al., 2020, pp. 30-33).

Asimismo, en cuanto a competencias transversales se pueden mencionar las siguientes:

- Comunicación oral y escrita en español e inglés para establecer una comunicación efectiva en situaciones culturalmente significantes.

- Manejo de información para seleccionar las fuentes de información relevantes y pertinentes según la situación, el problema o el proyecto, e integrar la base de conocimiento para sustentar argumentos y soluciones.

- Habilidades digitales para la producción, almacenamiento y recuperación de información, organización y visualización de datos, comunicación, creación, uso y gestión de espacios de interacción en medios digitales.

- Razonamiento ético y moral intrapersonal e interpersonal para la consideración razonada de la naturaleza de las normas o de los argumentos ético-morales y para desarrollar una reflexión crítica de las normas de conducta propias y sociales.

- Sostenibilidad para cubrir las necesidades económicas, sociales, de diversidad cultural, de un medio ambiente sano y de conservación y preservación.

- Metacognición para el conocimiento y regulación de los procesos cognitivos, es decir, para tomar conciencia del funcionamiento de su manera de aprender, comprender por qué los resultados de su actividad son positivos o negativos, planificar sus actividades e identificar estrategias y recursos para mejorar sus resultados (Fierro et al., 2020, pp. 30-33).

La validez de contenido de las variables de este instrumento conceptualiza como el nivel representación de cada ítem, que refleja un dominio específico de contenido (Hernández, Fernández y Baptista, 2014). Este se desarrolló a través del método de juicio de 


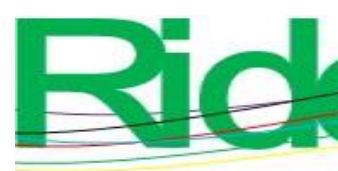

Revista Iberoamericana para la Investigación y el Desarrollo Educativo ISSN $2007-7467$

expertos, que define la opinión de personas con trayectoria en el tema, reconocidas por otros como profesionistas cualificados en el tópico a evaluar. La selección de los expertos son personas cuya especialización, experiencia profesional, académica o investigativa relacionada al tema de investigación les permite valorar cada uno de los ítems incluidos en la herramienta, la cual debe tener claridad en los objetivos y en el posicionamiento teórico (Soriano, 2014).

En la revisión de la literatura, Hernández-Sampieri y Mendoza (2018) describen la validez como el grado con que un instrumento mide la variable que pretende evaluar. Asimismo, Cohen y Swerdik (2020) mencionan que la validez del contenido denota qué tan conveniente es la muestra realizada a un universo según lo que se procura medir para designar como reactivos o ítems a los atributos de la población.

Otros autores — como Escobar-Pérez y Cuervo-Martínez (2008) — señalan que para redactar o definir se necesita una adecuada conceptualización y operacionalización del constructo, es decir, el investigador debe especificar previamente las dimensiones a medir y sus indicadores, a partir de los cuales se realizarán los ítems. La variable medida por el instrumento y el uso que se les dará a las puntuaciones obtenidas son aspectos fundamentales tanto para la estimación como para la conceptualización y la validez de contenido.

El proceso para hacer posible la validación de contenido del instrumento a través del juicio de expertos resulta eficiente cuando se especifica lo que se espera de ellos. Si bien este proceso puede realizarse través de diversos métodos, utilizar esta técnica tiene como resultado que el ítems o constructo garantice la rigurosidad científica del proceso (Pedrosa, Suárez-Álvarez y García-Cueto, 2013).

En el caso específico del coeficiente de confiabilidad, se vincula a la homogeneidad o consistencia interna de cada ítem, lo que se detalla a través del coeficiente del alfa propuesto por Lee J. Cronbach (1916-2001) en el año 1951. Este ha demostrado, desde el siglo pasado, que representa una generalización de las fórmulas KR-20 y KR-21 de consistencia interna desarrolladas en 1937 por Kuder y Richardson (Kerlinger y Howard, 2002), las cuales eran solo aplicables a formatos binarios de calificación o de respuestas dicotómicas. Por lo tanto, con la creación de la escala de Cronbach, los investigadores fueron capaces de evaluar la confiabilidad y consistencia interna de un instrumento constituido por una escala Likert o cualquier escala de opción múltiple. 

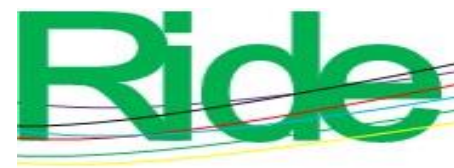

Revista Iberoamericana para la Investigación y el Desarrollo Educativo ISSN 2007 - 7467

Hernández, Fernández y Baptista (2014) explican que "la confiabilidad de un instrumento de medición se determina a través de diversas técnicas y, se refieren al grado en el cual su aplicación repetida al mismo sujeto de estudio, que producen resultados iguales” (p. 200). Al respecto, es importante diferenciar entre la fiabilidad y validez, como lo señala Morales (2007), ya que no son sinónimos, pues un instrumento puede ser válido al medir para lo que fue diseñado, pero sin la clasificación y orden de los sujetos de estudio.

En este sentido, debido a la reciente implementación del modelo educativo UACHDS, se carece de un instrumento que evalúe el perfil actitudinal del docente, así como las competencias universitarias y las transversales del docente de la Facultad de Odontología de la Universidad Autónoma de Chihuahua (UACH).

En revisión de investigaciones, se encontraron estudios similares, aunque existe un vacío del conocimiento en relación con este fenómeno de estudio. Por ende, surge la oportunidad de elaborar los instrumentos y validarlos en su contenido y confiabilidad, para lo cual se planteó la siguiente interrogante: ¿cuál es la validez de instrumento por juicio de expertos del perfil actitudinal del docente y su relación con el desarrollo de competencias universitarias y transversales?

Por consiguiente, en esta validación se planteó el siguiente objetivo: mostrar los resultados de la validación de contenido y consistencia interna de dos instrumentos que miden las siguientes variables:

- Variable independiente: Perfil actitudinal de los docentes de Odontología de la $\mathrm{UACH}$.

- Variable dependiente: Desarrollo de competencias universitarias y transversales (tabla 1). 
Tabla 1. Definición de variables

\begin{tabular}{|c|c|c|c|}
\hline Variables & Nominal & Conceptual & Operacional \\
\hline $\begin{array}{l}\text { Independiente } \\
\text { Perfil actitudinal } \\
\text { del docente }\end{array}$ & $\begin{array}{l}\text { Son aquellas } \\
\text { características más } \\
\text { importantes del } \\
\text { profesor como } \\
\text { facilitador del } \\
\text { proceso de } \\
\text { aprendizaje, es decir, } \\
\text { vinculación de la } \\
\text { teoría y la práctica, } \\
\text { clases dinámicas e } \\
\text { interactivas y uso de } \\
\text { experiencias reales } \\
\text { (Valerio y Rodríguez, } \\
\text { 2017). }\end{array}$ & $\begin{array}{l}\text { El perfil actitudinal } \\
\text { del docente } \\
\text { universitario se } \\
\text { define como } \\
\text { aquella persona que } \\
\text { manifiesta tres } \\
\text { grandes cualidades: } \\
\text { conocimiento } \\
\text { pedagógico, } \\
\text { habilidades } \\
\text { didácticas y valores } \\
\text { morales y éticos, } \\
\text { entre los que se } \\
\text { encuentran los } \\
\text { siguientes: } \\
\text { humanismo, } \\
\text { vocación, } \\
\text { responsabilidad, } \\
\text { compromiso y } \\
\text { conocimiento para } \\
\text { realizar una } \\
\text { planeación que se } \\
\text { pueda usar en } \\
\text { diversos contextos } \\
\text { y una evaluación } \\
\text { sistemática, } \\
\text { diversificada y justa } \\
\text { (Merellano-Navarro } \\
\text { Almonacid-Fierro, } \\
\text { Moreno-Doña y } \\
\text { Castro-Jaque 2016). }\end{array}$ & $\begin{array}{l}\text { Para la } \\
\text { operacionalización } \\
\text { del perfil actitudinal } \\
\text { del docente se } \\
\text { agruparon en } 38 \\
\text { variables, entre las } \\
\text { que se evaluaron lo } \\
\text { siguiente: ser } \\
\text { profesional, } \\
\text { respetuoso, } \\
\text { humanista } \\
\text { facilitador, } \\
\text { autocrático, entre } \\
\text { otros. Como } \\
\text { instrumento se } \\
\text { utilizó una escala } \\
\text { tipo Likert con } \\
\text { valores centesimales } \\
\text { con los siguientes } \\
\text { indicadores: } \\
\text { Univocidad óptima } \\
=3, \text { Univocidad alta } \\
=2, \text { Univocidad } \\
\text { baja, = 1 y } \\
\text { Univocidad nula = } \\
0 .\end{array}$ \\
\hline $\begin{array}{l}\text { Dependientes } \\
\text { Competencias } \\
\text { universitarias } \\
\text { Competencias } \\
\text { transversales }\end{array}$ & $\begin{array}{l}\text { Competencias } \\
\text { universitarias } \\
\text { trasversales } \\
\text { variables nominales } \\
\text { de tipo politómicas } \\
\text { que tienen tres o más } \\
\text { unidades que se van a } \\
\text { medir; en este caso, el } \\
\text { conjunto de atributos } \\
\text { que poseen los tipos } \\
\text { que las definen como } \\
\text { tal (Villasís-Keever y } \\
\text { Miranda-Novales, } \\
\text { 2016). }\end{array}$ & $\begin{array}{l}\text { Competencias } \\
\text { universitarias: } \\
\text { Son aquellos } \\
\text { conocimientos, } \\
\text { habilidades, } \\
\text { actitudes y valores } \\
\text { que todo estudiante } \\
\text { universitario debe } \\
\text { desarrollar; tienen } \\
\text { el propósito de } \\
\text { formarlo en las } \\
\text { categorías, } \\
\text { metodologías de } \\
\text { investigación y }\end{array}$ & $\begin{array}{lr}\text { Los aspectos por } \\
\text { evaluar incluyen las } \\
\text { variables de cada } \\
\text { una de las } \\
\text { competencias que } \\
\text { comprenden las } \\
\text { universitarias con } 45 \\
\text { ítems y 26 de las } \\
\text { competencias } \\
\text { transversales con los } \\
\text { siguientes } \\
\text { indicadores: } \\
\text { Univocidad óptima } \\
=3 \text {, Univocidad alta }\end{array}$ \\
\hline
\end{tabular}




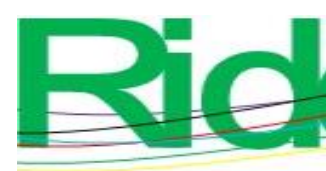

Revista Iberoamericana para la Investigación y el Desarrollo Educativo ISSN $2007-7467$

univocidad nula $=0$. Estuvo dirigido a docentes y estudiantes, con un total de 117 y 118 ítems, respectivamente, organizados en cuatro ejes principales: I) datos sociodemográficos, II) actitudes docentes, III) competencias universitarias y IV) competencias transversales, que se muestran en la tabla 2 .

Tabla 2. Estructura del instrumento

\begin{tabular}{|c|c|c|}
\hline Apartados & Ejes & Número de ítems \\
\hline I & Datos signalíticos & 8 \\
\hline II & Actitudes docentes & 38 \\
\hline III & Competencias universitarias & 45 \\
\hline IV & Competencias transversales & 26 \\
\hline & TOTAL & 117 \\
\hline
\end{tabular}

Fuente: Elaboración propia

Fase 2. La validación de contenido del instrumento se realizó a través de juicio de expertos, técnica que garantiza que el ítems o constructo responda a la rigurosidad científica del proceso (Pedrosa et al., 2013). El instrumento se envió a 10 docentes con trayectoria en investigación. La información emitida por cada uno de ellos se analizó a través del procedimiento definido por Carrera, Vaquero y Ballsels (2011) para determinar el índice de univocidad $\left(i_{U}\right)$ y pertinencia $\left(i_{P}\right)$, con la aplicación de las siguientes fórmulas:

$$
i_{U}=\frac{\left(\sum n_{U O} \cdot V_{U O}\right)+\left(\sum n_{U E} \cdot V_{U E}\right)+\left(\sum n_{U B} \cdot V_{U B}\right)+\left(\sum n_{U N} \cdot V_{U N}\right)}{\sum n_{T U} \cdot V_{M U}}
$$

Donde

${ }^{i} U=$ Índice de univocidad

$\sum=$ Sumatoria

$n_{U O}=$ Número de respuestas obtenidas del nivel de univocidad óptima

$V_{U O}=$ Valor asignado al nivel de univocidad

$n_{U E}=$ Número de respuestas obtenidas del nivel de univocidad elevada

$V_{U E}=$ Valor asignado al nivel de univocidad elevada 

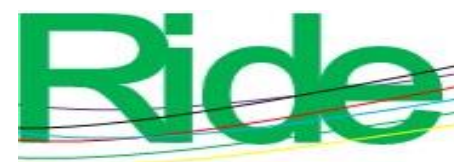

$n_{U B}=$ Número de respuestas obtenidas del nivel de univocidad baja

$V_{U B}=$ Valor asignado al nivel de univocidad baja

$n_{U N}=$ Número de respuestas obtenidas del nivel de univocidad nula

$V_{U N}=$ Valor asignado al nivel de univocidad nula

$n_{T U}=$ Número de respuestas totales obtenidas para el nivel de univocidad

$V_{M U}=$ Valor máximo de los niveles de la escala de univocidad

$$
i_{P}=\frac{\left(\sum n_{P O} \cdot V_{P O}\right)+\left(\sum n_{P E} \cdot V_{P E}\right)+\left(\sum n_{P B} \cdot V_{P B}\right)+\left(\sum n_{P N} \cdot V_{P N}\right)}{\sum n_{T P} \cdot V_{M P}}
$$

Donde

${ }_{P}^{i}=$ Índice de pertinencia

$n_{P O}=$ Número de respuestas obtenidas del nivel de pertinencia óptima

$V_{P O}=$ Valor asignado al nivel de pertinencia

$n_{P E}=$ Número de respuestas obtenidas del nivel de pertinencia elevada

$V_{P E}=$ Valor asignado al nivel de pertinencia baja

$n_{P B}=$ Número de respuestas obtenidas del nivel de pertinencia baja

$V_{P B}=$ Valor asignado al nivel de pertinencia baja

$n_{P N}=$ Número de respuestas obtenidas del nivel de pertinencia nula

$V_{P N}=$ Valor asignado al nivel de pertinencia nula

$n_{T P}=$ Número de respuestas totales obtenidas para el nivel de pertinencia

$V_{M P}=$ Valor máximo de los niveles de la escala de pertinencia

Fuente: Carrera, et al. (2011) 


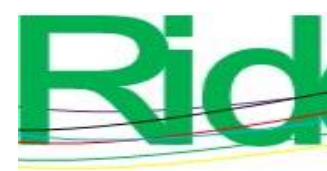

Revista Iberoamericana para la Investigación y el Desarrollo Educativo ISSN 2007 - 7467

La información arrojada por el piloteo se analizó con el programa estadístico Statistical Package for the Social Sciences (SPSS, v. 22).

\section{Resultados}

La revisión de los resultados por juicio de expertos en docencia permitió realizar el análisis de univocidad y pertinencia que se presenta a través de la siguiente tabla 5.

Tabla 5. Resultados de la validez del instrumento por jueces que determinan el índice de

\begin{tabular}{|l|l|l|l|}
\hline \multicolumn{2}{|c|}{ Resultados según $i_{U}$} & \multicolumn{2}{c|}{ Resultados según $i_{P}$} \\
\hline$i_{U} \geq .80$ & $\begin{array}{l}107 \text { ítems se mantienen en } \\
\text { su forma original. }\end{array}$ & $i_{P} \geq .80$ & $\begin{array}{l}110 \text { íms se mantienen en } \\
\text { su forma original. }\end{array}$ \\
\hline$i_{U} \leq .79 \geq .60$ & $\begin{array}{l}10 \text { ítems se modifican en } \\
\text { su redacción. }\end{array}$ & $i_{P} \leq .79 \geq .60$ & $\begin{array}{l}7 \text { ítems se modifican en su } \\
\text { redacción o ubicación. }\end{array}$ \\
\hline$i_{U} \leq .59$ & 0 ítems se eliminan. & $i_{P} \leq .59$ & 0 ítems se eliminan. \\
\hline
\end{tabular}

Fuente: Elaboración propia

En los índices de univocidad $\left(i_{U}\right)$ y pertinencia $\left(i_{P}\right), 109$ ítems conservaron el constructo original, 8 fueron modificados en su redacción y 3 se suprimieron. Es relevante considerar las recomendaciones propuestas por cuatro jueces expertos de ser eliminados; en este sentido, los resultados de la validez de los instrumentos de actitudes y competencias se presentan en la siguiente tabla 6. 
Tabla 6. Relación de los ítems modificados según el índice de univocidad y pertinencia

\begin{tabular}{|c|c|c|c|}
\hline Ítem original & $i_{U}$ & $I_{P}$ & Ítem modificado o eliminado \\
\hline Amigable & 0.8 & 0.733 & Cordial \\
\hline Autoritario(a) & 0.733 & 0.733 & Arbitrario (a) \\
\hline Conflictivo & 0.633 & 0.666 & Eliminado \\
\hline Impulsivo & 0.733 & 0.733 & Eliminado \\
\hline Inseguro & 0.7 & 0.666 & Eliminado \\
\hline Represivo & 0.7 & 0.666 & Restrictivo (a) \\
\hline $\begin{array}{l}\text { Identifique la problemática del } \\
\text { contexto real y la describa } \\
\text { mediante modelos } \\
\text { matemáticos. }\end{array}$ & 0.7667 & 0.8333 & $\begin{array}{l}\text { Identifique la problemática del contexto } \\
\text { real y la describa mediante ecuaciones } \\
\text { matemáticas }\end{array}$ \\
\hline $\begin{array}{l}\text { Modele procesos complejos, } \\
\text { evalúe datos, valore } \\
\text { probabilidades y evalúe } \\
\text { riesgos, prediga resultados o } \\
\text { modifique los procedimientos } \\
\text { para la resolución de } \\
\text { problemas de su entorno. }\end{array}$ & 0.7667 & 0.7667 & $\begin{array}{l}\text { Modele, evalúe y prediga resultados } \\
\text { para la resolución de problemas de su } \\
\text { entorno. }\end{array}$ \\
\hline $\begin{array}{l}\text { Distinga la investigación } \\
\text { social para profundizar en las } \\
\text { causas con información sobre } \\
\text { las opiniones, creencias y } \\
\text { valores de una sociedad en un } \\
\text { momento dado. }\end{array}$ & 0.7667 & 0.8667 & $\begin{array}{l}\text { Distinga la investigación social para } \\
\text { profundizar en las causas, opiniones, } \\
\text { creencias y valores de la sociedad. }\end{array}$ \\
\hline $\begin{array}{l}\text { Utilice las fuentes de } \\
\text { información para argumentar } \\
\text { y modelar explicaciones sobre } \\
\text { los fenómenos sociales e } \\
\text { históricos. }\end{array}$ & 0.7667 & 0.8667 & $\begin{array}{l}\text { Utilice las fuentes de información para } \\
\text { argumentar sobre fenómenos sociales e } \\
\text { históricos. }\end{array}$ \\
\hline $\begin{array}{l}\text { Perciba la estética en las } \\
\text { manifestaciones artísticas, } \\
\text { culturales y científicas para } \\
\text { mostrarla en sus expresiones } \\
\text { socioafectivas e intelectivas, } \\
\text { así como en las relaciones con } \\
\text { el otro y consigo mismo. }\end{array}$ & 0.7667 & 0.8667 & $\begin{array}{l}\text { Perciba la estética en las } \\
\text { manifestaciones artísticas y científicas } \\
\text { en sus expresiones afectivas e } \\
\text { intelectuales. }\end{array}$ \\
\hline
\end{tabular}

Fuente: Elaboración propia

El resultado del análisis de confiabilidad y consistencia en el cuestionario dirigido a los docentes mostró un alfa de Cronbach de a $=.972$, y para el instrumento de los estudiantes fue de a $=.989$ sin tener la necesidad de modificar ninguno de los ítems propuestos en este instrumento y se presenta en la tabla 7. 
Tabla 7. Validación del alfa de Cronbach del instrumento

\begin{tabular}{|c|c|c|c|c|}
\hline \multirow{2}{*}{ Casos } & \multicolumn{2}{|c|}{ Cuestionario para docentes } & \multicolumn{2}{c|}{ Cuestionario para estudiantes } \\
\cline { 2 - 5 } & $\mathrm{n}$ & $\%$ & $\mathrm{~N}$ & $\%$ \\
\hline Válido & 29 & 87.9 & 57 & 100 \\
\hline Excluido $^{\mathrm{a}}$ & 4 & 12.1 & 0 & 0 \\
\hline Total & 33 & 100.0 & 57 & 100 \\
\hline & $\begin{array}{c}\text { Alfa de } \\
\text { Cronbach }\end{array}$ & $\begin{array}{c}\mathrm{N} .^{\circ} \text { de } \\
\text { elementos }\end{array}$ & Alfa de Cronbach & \multirow{2}{*}{$\mathrm{N}^{\circ}{ }^{\circ}$ de elementos } \\
\cline { 2 - 5 } & .972 & 109 & .989 & 106 \\
\hline
\end{tabular}

Fuente: Elaboración propia

\section{Discusión}

La validación de los instrumentos que se presenta en este artículo permite evaluar el perfil actitudinal, las competencias universitarias y transversales del modelo educativo de la UACH (2020) que convergen en la formación de los estudiantes de Odontología. En tal sentido, se destaca la participación de juicio de expertos con sugerencias emitidas para cumplir con el propósito de este proceso metodológico y de investigación.

En relación con los resultados, se realizó la búsqueda de literatura vinculada a la validez de instrumentos. Al respecto, se puede mencionar una investigación de González y Suárez (2016) para la adaptación, validación y estandarización de cuestionarios para egresados y empleadores en el Estado de México. En tal sentido, una parte importante fue el ajuste del instrumento para que los ítems pudieran medir los objetos de estudio y las variables para las cuales se diseñó. De manera similar se adecuaron los constructos en relación con el perfil actitudinal del docente, las competencias universitarias y las transversales de los estudiantes de Odontología de la UACH.

Respecto a la validez por el juicio de expertos realizada en esta investigación a los instrumentos, los resultados del alfa de Cronbach de docentes fue de a $=.972$ y de los estudiantes de $\mathrm{a}=.989$, lo cual se encuentra en concordancia con los resultados de una doble valoración metodológica que realizó De la Cruz y Gordillo (2020), con una validación de univocidad y pertinencia a través del piloteo por juicio de expertos, con resultados de coherencia interna del alfa de Cronbach de $\geq 0.80$, por lo que se resalta el proceso de validación secuencial y metodológica que garantizó el proceso de validación por los resultados que arrojó un alto consenso por el juicio de expertos. 


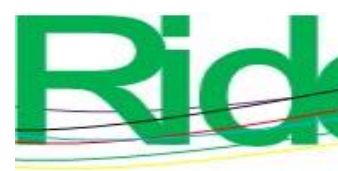

Revista Iberoamericana para la Investigación y el Desarrollo Educativo ISSN 2007 - 7467

En esta misma línea Sotelo, Sosa y Carreón (2020) validaron un instrumento para evaluar el desempeño de un sistema de gestión de calidad en una institución de educación superior. La obtención del alfa de Cronbach se efectuó por medio del cruce de varianza de cada ítem a través del software estadístico SPSS (v. 23), con un resultado de fiabilidad de .981 en 42 elementos; por ello, se tomó como referencia para este estudio, ya que arrojó resultados similares para establecer si el parámetro de fiabilidad era aceptable o no.

Otro estudio que cabe enfatizar es el que desarrollaron Parra, Garay y Aguilar (2019) para la validez y consistencia de un cuestionario que medía factores asociados a la salud, desempeño académico y profesional de los médicos residentes de México. Este cuestionario fue validado por juicio de expertos con un índice de univocidad y pertinencia de $\leq .79 \mathrm{y} \geq .60$. La medición del coeficiente del alfa de Cronbach fue de $\mathrm{a}=.820$, resultados que se consideran como referentes teóricos para modificar constructos.

En tal sentido, se optó por transformar ocho ítems del presente instrumento $(10,11$, 42, 58, 62, 64, 71 y 74) y se eliminaron las preguntas 18, 31 y 33 para mejor la redacción y comprensión por parte de los participantes.

\section{Conclusiones}

Como se manifiesta en los resultados de esta investigación, los instrumentos reflejan un buen nivel de validación de univocidad, pertinencia y consistencia interna, así como del análisis estadístico por medio del alfa de Cronbach de las competencias universitarias y transversales propuestas en el modelo educativo de la UACH (2020).

En este contexto, el proceso de validación de los instrumentos permitió exponer los resultados de un proceso articulado y con rigor científico. En consecuencia, se puede asegurar que se logró el objetivo planteado, pues se garantizó el éxito mediante el análisis metodológico y sistemático que favoreció la validación de constructos a través de este proceso. 


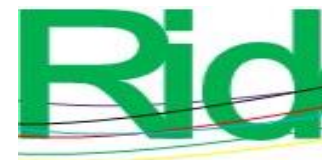

Revista Iberoamericana para la
Investigación y el Desarrollo Educativo
ISSN $2007-7467$

\section{Futuras líneas de investigación}

Los resultados presentan un marco de referencia para contribuir a la generación de conocimiento mediante la realización de proyectos de investigación en distintas líneas o con otras disciplinas bajo el enfoque cuantitativo y educativo, ya que la trascendencia y rigurosidad del proceso de validación garantiza la obtención real y confiable de la información.

\section{Limitaciones y fortalezas del estudio}

Las instituciones universitarias están en constante renovación, y la UACH no es la excepción; por consiguiente, el instrumento validado procuró recabar información de un modelo educativo en construcción para evaluar el desarrollo de las competencias universitarias y trasversales de los estudiantes y docentes de la disciplina de Odontología con la finalidad de identificar áreas de oportunidad y aportar elementos para considerar en la estructura, organización y ejecución. Esta investigación, en pocas palabras, ofrece a los investigadores el procedimiento para la validación de un instrumento de tipo cuantitativo que pudiera ser usado en otros contextos, aunque con los ajustes que sean requeridos por cada contexto. 

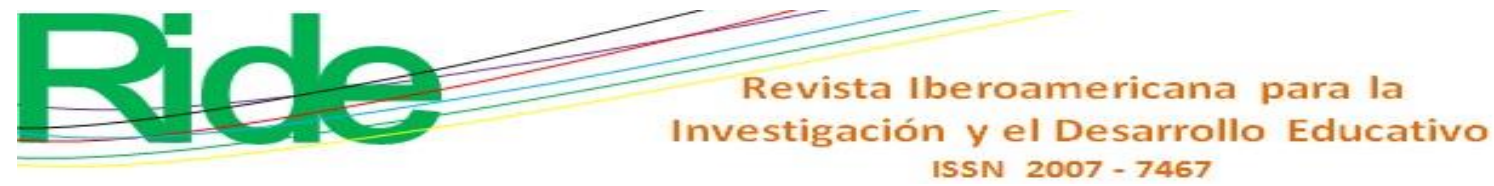

\section{Referencias}

Carrera, F. F., Vaquero, E. T. y Balsells, M. B. (2011). Instrumento de evaluación de competencias digitales para adolescentes en riesgo social. Revista Electrónica de Tecnología Educativa, (35), 1-25.

Cohen, R. y Swerdlik, M. (2020). Pruebas y evaluación psicológicas: introducción a las pruebas y a la medición (6. ${ }^{\mathrm{a}}$ ed.). México: Mc-Graw-Hill Interamericana.

De la Cruz, V. y Gordillo, E. (2020). Validación de entrevistas por juicio de expertos en el estudio de la inclusión educativa en el área de lenguas extranjeras. Revista Iberoamericana para la Investigación y el Desarrollo Educativo, 11(21). Doi: https://doi.org/10.23913/ride.v11i21.710

Escobar-Pérez, J. y Cuervo-Martínez, A. (2008). Validez de contenido y juicio de expertos: una aproximación a su utilización. Avances en Medición, 6, 27-36. Recuperado de https://www.researchgate.net/profile/Jazmine_Escobar-

Perez/publication/302438451_Validez_de_contenido_y_juicio_de_expertos_Una_a proximacion_a_su_utilizacion/links/59a8daecaca27202ed5f593a/Validez-decontenido-y-juicio-de-expertos-Una-aproximación-a-su-utilizacion.pdf

Fierro R, LA., Basurto S, M., Guzmán I, I., Hernández O, G., Lara A, J., Marín U, R., Parra A, H., Sáenz L, E., Torres P, R., Trejo A. AB., Flores, V. MA., (2020). Modelo educativo para el desarrollo sostenible. Universidad Autónoma de Chihuahua. Recuperado de https://faciatec.uach.mx/facultad/nuevo-modelo-educativo-uach-ds/

González, E. y Suárez, E. (2016). Adaptación, validación y estandarización de cuestionarios para egresados y empleadores en el Estado de México. Revista Iberoamericana para la Investigación y el Desarrollo Educativo, 6(12). Recuperado de https://www.ride.org.mx/index.php/RIDE/article/view/222/1000

Hernández, R., Fernández, C. y Baptista, P. (2014). Metodología de la investigación (6. ${ }^{\mathrm{a}}$ ed.).

México D. F.: McGraw-Hill. Interamericana Editores, S. A. de C. V.

Hernández-Sampieri, R. y Mendoza, T. C. P. (2018). Metodología de la investigación (6. ${ }^{\mathrm{a}}$ ed.). México D. F.: Mc-Graw-Hill Interamericana.

Kerlinger, F. N. y Howard, R. L. (2002). Investigación del comportamiento. Métodos de Investigación en ciencias sociales. México: Mc Graw-Hill Interamericana. 


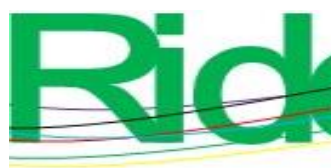

Revista Iberoamericana para la Investigación y el Desarrollo Educativo ISSN 2007 - 7467

Merellano, A., Almonacid, A., Moreno, C. y Castro, C. (2016). Buenos docentes universitarios: ¿qué dicen los estudiantes? Revista Educ. Pesqui., 42(4), 937-952. Doi: http://dx.doi.org/10.1590/S1517-9702201612152689

Morales, P. (2007). La fiabilidad de los test y escalas. Madrid: Universidad Pontificia Comillas. Facultad de Ciencias Humanas y Sociales. Recuperado de https://matcris5.files.wordpress.com/2014/04/fiabilidad-tests-y-escalas-morales2007.pdf

Parra, A., Garay, J. y Aguilar E. (2019). Validez y consistencia de un cuestionario que mide: factores asociados a la salud, desempeño académico y profesional de los médicos residentes de México. RECIE. Revista Electrónica Científica de Investigación Educativa, 4(2), 1327-1336.

Pedrosa, I., Suárez-Álvarez, J. y García-Cueto, E. (2013). Evidencias sobre la validez de contenido: avances teóricos y métodos para su estimación. Acción Psicológica, 10(2), 3-18. Doi: https://dx.doi.org/10.5944/ap.10.2.11820

Soriano, A. M. (2014). Diseño y validación de instrumentos de medición. Diá-logos, 14, 1940. Recuperado de http://redicces.org.sv/jspui/bitstream/10972/2105/1/2\%20disenoyvalidacion_dialogos 14.pdf

Sotelo, J. G., Sosa, M. C. y Carreón, E. (2020). Validación del instrumento de evaluación de desempeño de un sistema de gestión de calidad en una institución de educación superior. RIDE Revista Iberoamericana para la Investigación y el Desarrollo Educativo, 10(20). Doi: https://doi.org/10.23913/ride.v10i20.653

Valerio, U. G. y Rodríguez, M. D. C. (2017). Perfil del profesor universitario desde la perspectiva del estudiante. Innovación Educativa, 17(74), 109-124.

Villasís-Keever, M. Á. y Miranda-Novales, M. G. (2016). El protocolo de investigación IV: las variables de estudio. Revista Alergia México, 63(3). 


\begin{tabular}{|l|l|}
\hline Rol de contribución & Autor(es) \\
\hline Conceptualización & $\begin{array}{l}\text { Josué Israel Espinoza Solís - igual Norma Pizarro - igual } \\
\text { Haydeé Parra Acosta - igual Eliazar González Carrillo - } \\
\text { igual - Oscar Joel Talavera Sánchez - igual Gerardo } \\
\text { Bueno Acuña - igual }\end{array}$ \\
\hline Metodología & $\begin{array}{l}\text { Josué Israel Espinoza Solís - igual } \\
\text { Eliazar González Carrillo - igual }\end{array}$ \\
\hline Investigación & $\begin{array}{l}\text { Josué Israel Espinoza Solís - igual } \\
\text { Norma Pizarro - igual } \\
\text { Haydeé Parra Acosta - igual } \\
\text { Eliazar González Carrillo - igual } \\
\text { Oscar Joel Talavera Sánchez - igual } \\
\text { Gerardo Bueno Acuña - igual }\end{array}$ \\
\hline Análisis formal & $\begin{array}{l}\text { Josué Israel Espinoza Solís - igual } \\
\text { Oscar Joel Talavera Sánchez - igual }\end{array}$ \\
\hline Curación de datos & $\begin{array}{l}\text { Josué Israel Espinoza Solís - igual } \\
\text { Oscar Joel Talavera Sánchez - igual }\end{array}$ \\
\hline $\begin{array}{l}\text { Escritura, preparación del } \\
\text { borrador original }\end{array}$ & $\begin{array}{l}\text { Norma Pizarro - principal } \\
\text { Josué Israel Espinoza Solís - apoyo }\end{array}$ \\
\hline Escritura, revisión y edición & $\begin{array}{l}\text { Norma Pizarro - igual } \\
\text { Josué Israel Espinoza Solís }\end{array}$ \\
\hline Supervisión & Norma Pizarro \\
\hline Asesoría de proyectos & Josué Israel Espinosa Solís \\
\hline Adquisición de fondos & Gerardo Bueno Acuña \\
\hline
\end{tabular}

